\section{Ocorrência de parasitas com potencial zoonótico em fezes de cães coletadas em áreas públicas do município de Ribeirão Preto, SP, Brasil}

\section{Parasites with zoonotic potential in dog feces collected from public areas of the city of Ribeirão Preto, SP, Brazil}

\section{Resumo}

Objetivo: Avaliar a ocorrência de parasitos zoonóticos em fezes de cães colhidas em áreas públicas do município de Ribeirão Preto, enfatizando o risco da ocorrência de infecção humana. Métodos: Entre os meses de maio a dezembro de 2003 foram visitadas 78 praças, localizadas em cinco diferentes áreas do município: sudoeste, noroeste, norte, central e sudeste. Foram recolhidos 331 "pools" de material fecal canino, os quais foram processados pela técnica de sedimentação espontânea. Resultados: Em 56,8\% "pools" foram observados os parasitos zoonóticos: Ancylostoma spp. (41,7\%), Toxocara canis (24,2\%), Trichuris vulpis (15,7\%), Giardia spp. (10,2\%) e Isospora spp. (3,3\%). A prevalência de parasitos foi maior nas praças localizadas na área norte do município (70\%). Conclusões: É necessária a adoção de programas de preservação sanitária destes locais, no sentido de prevenir a contaminação ambiental por parasitos potencialmente patogênicos para o homem.

Palavras-chave: Parasitos zoonóticos. Contaminação. Fezes. Praças públicas. Cães.

\section{Divani Maria Capuano' \\ Gutemberg de Melo Rocha² \\ 'Seção de Biologia Médica do Instituto Adolfo Lutz - Laboratório I de Ribeirão Preto. \\ ${ }^{2}$ Departamento de Biologia Celular, Molecular e Bioagentes Patogênicos da Faculdade de Medicina de Ribeirão Preto - Campus da USP.}

Trabalho realizado no Departamento de Biologia Celular, Molecular e Bioagentes Patogênicos da Faculdade de Medicina de Ribeirão Preto - USP

Correspondência: Divani M. Capuano. Instituto Adolfo Lutz, Laboratório I de Ribeirão Preto, Rua Minas, 877, Campos Elíseos, 14085-410 Ribeirão Preto, São Paulo, Brasil. E-mail: dcapuano@ial.sp.gov.br 
Abstract

Objective: To assess the occurrence of zoonotic parasites in dog feces collected in public areas of the city of Ribeirão Preto, to alert to the risk of human infection. Methods: From May to December, 2003, 78 public squares located in five different areas of the city were visited: southwest, northwest, north, central and southeast. We collected 331 fecal canine pools that were processed by the spontaneous sedimentation technique. Results: $56.8 \%$ of pools presented zoonotic parasites such as: Ancylostoma spp. (41.7\%), Toxocara canis (24.2\%), Trichuris vulpis (15.7\%), Giardia spp. (10.2\%) and Isospora spp. (3.3\%). The prevalence of parasites was higher in squares located in the north area of the city (70\%). Conclusions: The adoption of sanitary preservation programs in these sites is necessary in order to prevent environmental contamination by parasites potentially pathogenic to man.

Keywords: Zoonotic parasites. Contamination. Feces. Public squares. Dogs.

\section{Introdução}

O papel do cão como um hospedeiro definitivo de várias parasitoses com potencial zoónotico tem sido largamente estudado e reconhecido como um importante problema de saúde pública. A crescente aquisição de cães como animais de companhia tem aumentado o número de pessoas expostas ao risco de contrair infecções por parasitos zoonóticos. Contudo, o risco de contaminação humana não está limitado apenas ao âmbito doméstico, pois, freqüentemente, os cães são levados por seus proprietários para passear em áreas públicas destinadas à recreação humana. Se os animais parasitados defecarem nestes locais podem contaminar o meio ambiente, sendo que o contato da população que freqüenta estas áreas com as fezes dos cães ou com o solo contaminado pode favorecer a infecção humana. A população infantil corresponde ao grupo mais exposto devido ao hábito de brincar em contato com o solo e aos distúrbios de perversão do apetite como a geofagia. Por outro lado, o crescimento urbano, com o estabelecimento de novas comunidades e conjuntos habitacionais, leva tanto ao aumento da população de cães de estimação como de cães errantes. Do ponto de vista epidemiológico, os cães errantes têm um papel importante na contaminação do meio ambiente, pois o fato de não receberem tratamento antiparasitário, aliado à facilidade com que circulam por várias áreas públicas, favorece a disseminação de enteroparasitas. Vários autores já assinalaram a presença de parasitos com potencial zoonótico em cães, através de necrópsia ou do exame de fezes, em estudos realizados em diversos municípios do Brasil ${ }^{1-6,16-18}$. Entretanto, são escassos os trabalhos avaliando a presença de parasitos em fezes colhidas em áreas públicas ${ }^{7-11}$.

O presente estudo teve como objetivo avaliar a ocorrência de parasitos caninos de potencial zoonótico em praças públicas do município de Ribeirão Preto, enfatizando o risco de infecção humana. 


\section{Métodos}

\section{Escolha da amostra}

Ribeirão Preto, incluindo o distrito de Bonfim Paulista, possui 160 praças semi ou totalmente urbanizadas, segundo cadastro do Setor de Parques e Jardins do município. A amostragem deste estudo foi constituída por 78 praças, sendo 67 sorteadas aleatoriamente e 11 incluídas na pesquisa por possuírem áreas de recreação infantil. Para o cálculo do tamanho da amostra, considerou-se um nível de confiança de 95\% . As praças selecionadas localizam-se em cinco diferentes áreas do município: sudoeste, noroeste, norte, central e sudeste, delimitadas segundo critérios estabelecidos pela Secretaria Municipal da Saúde de Ribeirão Preto.

\section{Considerações Éticas}

Este estudo foi aprovado pelo Comitê de Ética em Pesquisa do Instituto Adolfo Lutz.

\section{Coleta e processamento das amostras fecais caninas}

Em cada praça selecionada foram estabelecidos cinco pontos diferentes de coleta: quatro laterais externos e um ponto central. Entre os meses de maio a dezembro de 2003, em cada ponto das praças foram recolhidas entre uma e três amostras fecais de cães presentes no solo ou na grama. Em cada praça as amostras foram colhidas em sacos plásticos limpos, formando-se assim um "pool”. As fezes ressecadas ou deterioradas não foram colhidas. Os "pools" foram mantidos em isopor com gelo até a chegada ao laboratório, onde, após cuidadosa homogeneização, foram imediatamente processados pela técnica de sedimentação espontânea. Parte do sedimento obtido foi colocado sobre uma lâmina, adicionado de lugol e analisado em microscópio óptico com objetivas de $10 \mathrm{X}$ e 40X. Foram realizadas duas obser- vações microscópicas: após 2 horas e após 16 horas de sedimentação. Os ovos de Trichuris foram medidos sob ocular micrométrica para a distinção entre $T$. trichiura e T. vulpis. A identificação das larvas de Ancylostoma spp. foi baseada nas características morfométricas das mesmas.

\section{Análise estatística}

O teste de qui-quadrado foi utilizado para avaliar a associação entre a prevalência de parasitos encontrados nas praças investigadas, de acordo com as áreas do município. Foram considerados estatisticamente significante os valores de $\mathrm{p}<0,05$.

\section{Resultados}

Foram identificadas formas de parasitos zoonóticos em 56,8\% (188/331) das amostras avaliadas. Em 26,0\% (86/331) das amostras foi encontrada apenas uma espécie de parasito, enquanto que em $30,8 \%$ $(102 / 331)$ até três parasitos diferentes. As associações mais freqüentemente observadas foram: Ancylostoma spp. e Toxocara canis (27,4\%), Ancylostoma spp. e Trichuris vulpis (24,5\%), Ancylostoma spp., Toxocara canis e Giardia spp. (14,7\%) e Toxocara canis e Trichuris vulpis (12,7\%).

A freqüência dos diferentes parasitos encontrados está apresentada na Figura 1. Dos 41,7\% (138/331) “pools” com Ancylostoma spp., 35,5\% (49/138) apresentavam larvas rabditóides e filarióides. Dentre os 24,2\% (80/331) "pools" com ovos de T. canis, $21,2 \%$ (17/80) estavam embrionados, em fase de mórula ou larvados. A Tabela 1 demonstra a distribuição das praças contaminadas por parasitos zoonóticos, de acordo com as áreas do município.

\section{Discussão}

O nematódeo mais freqüentemente encontrado neste estudo foi Ancylostoma spp. $(41,7 \%)$, semelhantemente aos resultados obtidos por Castro et al. ${ }^{8}$ no município de Praia Grande, SP, Martins et al. ${ }^{9}$ em 
Tabela 1 - Distribuição de praças públicas contaminadas com parasitas zoonóticos, de acordo com as áreas do município de Ribeirão Preto, SP, Brasil.

Table 1 - Distribution of public squares contaminated by zoonotic parasites according to the areas of Ribeirão Preto, SP, Brazil.

\begin{tabular}{lcccc}
\hline & \multicolumn{4}{c}{ PRAÇAS PÚBLICAS } \\
\cline { 2 - 5 } ÁREA & Existentes & Investigadas & $\mathrm{N}$ & $\%$ \\
\hline Sudoeste & 13 & 7 & 4 & 57,0 \\
Noroeste & 17 & 10 & 6 & 60,0 \\
Norte & 22 & 10 & 7 & 70,0 \\
Central & 53 & 25 & 8 & 32,0 \\
Sudeste & 55 & 26 & 12 & 46,0 \\
\hline Total & 160 & 78 & 37 & 47,0 \\
\hline
\end{tabular}

$\chi^{2}=33.9(4 \mathrm{gl}) ; \mathrm{p}=0,0000$

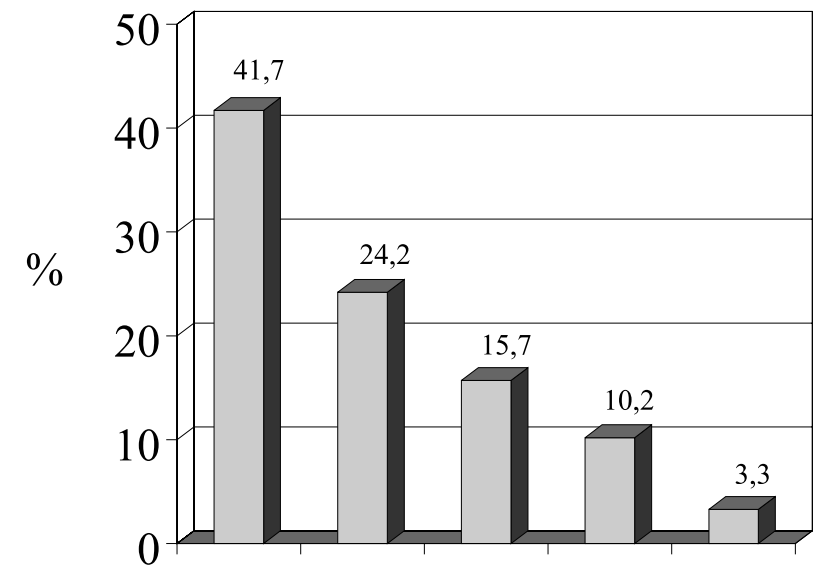

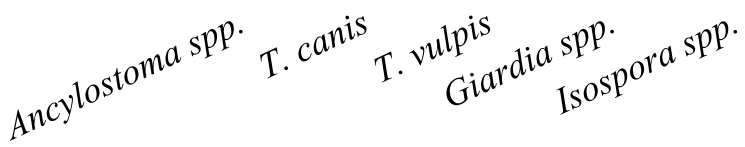

Figura 1 - Freqüência de parasitos com potencial zoonótico presentes em 331 "pools" de fezes colhidos em praças públicas do município de Ribeirão Preto, SP, Brasil.

Figure 1 - Frequency of parasites with zoonotic potential in 331 dog feces pools collected in public squares of the city of Ribeirão Preto, São Paulo, Brazil.

Manaus, AM e Scaini et al. ${ }^{10}$ no Balneário Cassino, RS, com prevalências de 45,9\%, $50,9 \%$ e $71,3 \%$, respectivamente. Este resultado é esperado, pois os cães podem ser parasitados pelos ancilostomídeos por toda a vida, sendo que desenvolvem na maturidade, imunidade contra os ascarídeos ${ }^{13}$. O segundo parasito mais observado foi $T$. canis (24,2\%), seguido pelo T. vulpis (15,7\%). Estes resultados não refletem os encontrados por Martins et al. ${ }^{9}$ em Manaus, AM, com prevalências de 16,8\% para T. vulpis e 6,8\% para T. canis e por Scaini et al. ${ }^{10}$ no Balneário Cassino, RS, que constatou freqüências de $32,5 \%$ para $T$. vulpis e de $9,3 \%$ para $T$. canis. A positividade de T.canis observada neste estudo foi maior do que a obtida por Alcântara et al. ${ }^{7}$, em Salvador, BA, de 18,4\% e por Castro et al. ${ }^{8}$ no município de Praia Grande, SP, de 1,2\%.

O encontro de larvas de Ancylostoma spp. e de ovos embrionados de $T$. canis, 
tem uma importância epidemiológica significante, indicando a existência de condições ambientais favoráveis ao desenvolvimento deste parasitas, o que representa um risco para a saúde pública, devido a possibilidade da ocorrência da Larva Migrans Cutânea (LMC) e da Larva Migrans Visceral (LMV) ${ }^{12,13}$.

Apesar das duas espécies, T. vulpis do cão e $T$. trichiura do homem, possuírem uma alta especificidade de hospedeiros, já foram assinalados casos de infecção entérica e de síndrome da Larva Migrans Visceral humanas pela espécie canina ${ }^{14,15}$. Como os ovos de ambas as espécies possuem similaridade morfológica, a correta identificação de $T$. vulpis no material fecal humano e canino é importante para estabelecer o cão como a fonte de infecção, realizar o tratamento antiparasitário do animal e prevenir a transmissão humana.

A prevalência de Giardia spp. observada neste estudo de $10,2 \%$, foi mais elevada do que a obtida por Martins et al. ${ }^{9}$ em Manaus, AM, de $0,6 \%$. Os cães podem estar parasitados por cepas de Giardia potencialmente infectantes para o homem ${ }^{12,17}$. Contudo, a giardíase nos cães geralmente é assintomática, o que dificulta o estabelecimento do diagnóstico desta parasitose nos animais, que passam a ser uma importante fonte de eliminação de cistos no meio ambiente, podendo contaminar o homem e outros animais ${ }^{12,13}$.

Isospora spp. é um protozoário patogênico que pode ser transmitido dos animais domésticos para o homem, através da água e alimentos contaminados com os oocistos, a forma infectante do parasita ${ }^{12,13}$. A infecção no homem causa desconforto gastrointestinal e diarréia crônica ${ }^{12,13}$. Este parasito, observado com uma freqüência de 3,3\% neste estudo, não foi encontrado por outros autores em trabalhos semelhantes $^{8-10}$. Entretanto, levantamentos baseados nos exames coprológicos de cães realizados em diversos municípios do Brasil, revelaram prevalências de isosporíase entre $0,38 \%$ a $8,5 \%$ e de giardíase entre $7,7 \%$ a $38 \%$, indicando que estes proto- zoários são freqüentes nos cães ${ }^{1-6,16-18}$. Oliveira-Sequeira et al. ${ }^{5}$, examinando 271 amostras de fezes de cães em Botucatu, SP, constataram maior eficácia do método de centrífugo-flotação em relação aos métodos de sedimentação e de flotação simples, na detecção de Giardia spp. e Isospora spp. Portanto, as freqüências destes parasitos neste estudo podem estar subestimadas devido a não utilização de técnicas de flutuação, mais sensíveis para a detecção de protozoários.

Este estudo permitiu avaliar a distribuição da ocorrência de parasitos zoonóticos nas praças localizadas em áreas do município com diferentes condições socioeconômicas. Verificou-se uma maior prevalência de parasitos nas praças localizadas na área norte do município $\left(\chi^{2}=33.9 ; \mathrm{p}=\right.$ $0,0000)$. Esta área situa-se numa região mais periférica do município, onde estão localizados vários conjuntos habitacionais destinados à população de baixa renda, bem como algumas favelas. Estudos conduzidos na Argentina por Sánchez-Thenevet et al. ${ }^{19}$ na Província de Chubut, na Patagônia. e por Andresuik et al. ${ }^{20}$ na cidade de Mar del Plata, demonstraram que um maior grau de contaminação ambiental por parasitos zoonóticos estava relacionado às baixas condições socioeconômicas e culturais da população destes locais.

\section{Conclusões}

Apesar de ter sido empregado apenas um método diagnóstico, e de examinarmos "pools" e não amostras fecais isoladas neste estudo, os resultados obtidos demonstram pioneiramente a presença de parasitos zoonóticos em praças públicas do município de Ribeirão Preto, indicando a existência de um problema de saúde pública. Esperamos que estes resultados possam servir de subsídios para que as autoridades competentes implementem programas sanitários como o controle de cães errantes, a promulgação de leis para evitar o acesso de cães em áreas públicas e a promoção de educação em saúde da 
população em geral, no sentido de prevenir a contaminação das praças públicas por parasitos patogênicos para o homem.

\section{Agradecimentos}

Ao Prof. Dr. Amaury Lelis Dal Fabbro do Departamento de Medicina Social da Faculdade de Medicina de Ribeirão Preto, pela orientação estatística, e à Sra. Eloísa Fonseca Del Tedesco, da Seção de Biologia Médica do Instituto Adolfo Lutz de Ribeirão Preto, pelo apoio na realização deste estudo.

\section{Referências}

1. Carollo MCC, Amato Neto V, Braz LMA, Kim DW. Pesquisa de oocistos de Cyclospora sp em fezes de cães da Grande São Paulo, Estado de São Paulo, Brasil. Rev Soc Bras Med Trop 2001; 34: 597-8.

2. Gennari SM, Kasai N, Pena HFJ, Cortez A. Ocorrência de protozoários e helmintos em amostras de fezes de cães e gatos da cidade de São Paulo. Braz J Vet Res Anim Sci 1999; 36: 87-91.

3. Hoffmann NA, Beltrão N, Botton AS, Caminha BX, Rue ML. Intestinal nematodes of stray dogs as zoonoses agents in D. Pedrito city (RS-Brazil). Bol Chil Parasitol 2000; 55: 92-3.

4. Blazius RD, Emerick S, Prophiro JS, Romão PRT, Silva OS. Ocorrência de protozoários e helmintos em amostras de fezes de cães errantes da cidade de Itapema, Santa Catarina. Rev Soc Bras Med Trop 2005; 38: 73-4.

5. Oliveira-Sequeira TC, Amarante AF, Ferrari TB, Nunes LC. Prevalence of intestinal parasites in dogs from São Paulo State, Brazil. Vet Parasitol 2002; 103: 19-27.

6. Leite LC, Marinoni LP, Círio SM, Diniz JMF, Silva MAN, Luz E et al. Endoparasitas em cães (Canis familiaris) na cidade de Curitiba - Paraná - Brasil. Arch Vet Sci 2004; 9 : 95-9.

7. Alcântara N, Bavia E, Silvão RM, Carvalho E. Environmental contamination by Toxocara sp eggs in public areas of Salvador, Bahia State, Brazil. Rev Soc Bras Med Trop 1989; 22: 187-90.

8. Castro JM, Santos SV, Monteiro NA. Contaminação de canteiros da orla marítima do município de Praia Grande, São Paulo, por ovos de Ancylostoma e Toxocara em fezes de cães. Rev Soc Bras Med Trop 2005; 38: 199-201.

9. Martins M, Soares AR, Moura MAS, Chaves AC, Silva RS, Barros JA. Levantamento de Toxocara canis no município de Manaus-AM. Dados preliminares. Rev Soc Bras Med Trop 2003; 36 (S1): 194-5.

10. Scaini CJ, Toledo RN, Lovatel R, Dionello MA, Gatti FA, Susin L et al. Contaminação ambiental por ovos e larvas de helmintos em fezes de cães na área central do Balneário Cassino, Rio Grande do Sul. Rev Soc Bras Med Trop 2003; 36: 617-9.
11. Araújo FR, Crocci AJ, Rodrigues RGC, Avalhaes JS, Miyoshi MI, Salgado FP et al. Contaminação de praça públicas de Campo Grande, Mato Grosso do Sul, Brasil, por ovos de Toxocara e Ancylostoma em fezes de cães. Rev Soc Bras Med Trop 1999; 32: 581-3.

12. Robertson D, Irwin PJ, Lymbery AJ, Thompson RCA. The role of companion animals in the emergence of parasitic zoonoses. Int J Parasitol 2000; 30: 1369-77.

13. Tan JS. Human zoonotic infections transmitted by dogs and cats. Arch Intern Med 1997; 157: 1933-43.

14. Dunn JJ, Columbus ST, Aldeen WE, Davis M, Carroll, KC. Trichuris vulpis recovered from a patient with chronic diarrhea and five dogs. J Clin Microbiol 2002; 40: 2703-4.

15. Sakano T, Hamamoto K, Kobayashi TM, Usui T. Visceral larva migrans caused by Trichuris vulpis. Arch Dis Child 1980; 55: 631-3.

16. Bartmann A. Freqüência de Giardia lamblia (Kunstler, 1882), em cães (Canis familiaris) determinada através de exames parasitológicos solicitados por clínicas veterinárias da cidade de Porto Alegre, Rio Grande do Sul, Brasil. Porto Alegre, 2002 [dissertação de mestrado]. Porto Alegre: Universidade Federal do Rio Grande do Sul; 2002.

17. Sogayar MIL, Corrêa FMA. Giardia in dogs in Botucatu, São Paulo State, Brazil: a comparative study of canine and human species. Rev Cienc Biomed São Paulo 1984; 5: 69-73.

18. Beck C, Araújo FAP, Olicheski AT, Breyer AS. Freqüência da infecção por Giardia lamblia (Kunstler, 1882) em cães (Canis familiaris) avaliada pelo Método de Faust e cols. (1939) e pela Coloração da Auramina, no município de Canoas, RS, Brasil. Cienc Rural 2005; 35: 126-30.

19. Sánchez-Thevenet P, Jensen O, Mellado I, Torrecillas C, Raso J, Flores ME et al. Presence and persistence of intestinal parasites in canine fecal material collected from the environment in the Province of Chubut, Argentine Patagonia. Vet Parasitol 2003; 117: 263-9.

20. Andresiuk MV, Rodríguez F, Denegri GM, Sardella NH, Hollmann P. Relevamiento de parásitos zoonóticos en materia fecal canina y su importancia para la salud de los niños. Arch Argent Pediatr 2004; 102: 325-9.

Recebido em: 29/04/05

Versão reformulada reapresentada em: 16/01/06 Aprovado em: 20/01/06 